\title{
Granulation with indistinguishability, equivalence, or similarity
}

\author{
C. Maria Keet \\ Faculty of Computer Science, Free University of Bozen-Bolzano \\ Piazza Domenicani 3, 39100 Bolzano, Italy. keet@inf .unibz. it
}

\begin{abstract}
One of the relations used with granularity is indistinguishability, where distinguishable entities in a finer-grained granule are indistinguishable in a coarser-grained granule. This relation is a subtype of equivalence relation, which is used in the other direction to create finer-grained granules. Together with the notion of similarity, we formally prove some intuitive properties of the indistinguishability relation for both qualitative and quantitative granularity, that with a given granulation there must be at least two granules (levels of granularity) for it to be granular, and derive a strict order between finer and coarser granules. Based on these results, granulation hierarchy is defined as extra assisting structure to augment implementations.
\end{abstract}

\section{INTRODUCTION}

Similarity, indistinguishability, and equivalence are essential relations in granular computing, which are related but not interchangeable. The differences - and consequences following from them-are salient when the intended use is examined not only for quantitative granular computing, but, moreover, for qualitative aspects (semantics) of granularity.

The comprehensive description of granule and granulation by Zadeh [1] is useful for grouping together several notions about granular computing: "Informally, granulation of an object $A$ results in a collection of granules of $A$, with a granule being a clump of objects (or points) which are drawn together by indistinguishability, similarity, proximity or functionality... In general, granulation is hierarchical in nature.". The similarity and equivalence relations have been well investigated with set-based approaches (e.g., [2]-[8]). Comparatively little effort has gone into indistinguishability [9]-[11], the differences between the three relations, and the interplay between them. With this contribution we aim to specify their differences more precisely, thereby giving a semanticsbased, formal underpinning where we take into account both quantitative and qualitative notions (also called scale-based and non-scale semantics or data values and knowledge [12], [13]). It will be demonstrated that the indistinguishability relation is a special type of the equivalence relation, because it has more constraints, and we demonstrate the difference in intended usage: generalising versus specialising, respectively. The indistinguishability relation is reflexive, symmetric and transitive. Further, by availing of similarity as well, we prove that there must be at least two 'levels of granularity' in a hierarchy - a fine-grained and an adjacent coarse-grained granule - and not three as was put forward by [14], [15]. Last, from the proofs it also follows that there has to be a strict order between fine- and coarse-grained granules.
The remainder of this paper is structured as follows. In section II we revisit the similarity relation, for both quantitative and qualitative aspects. Section III goes into detail on indistinguishablity, indiscernibility, and equivalence. The indistinguishability definition and proofs are in section IV and we conclude in section $\mathrm{V}$.

\section{Similarity}

Similarity emphasises why universals (types, classes) or particulars (instances) reside in the same granule, or not, and consequently provide a rationale for allocating entities and instances to a particular granule ${ }^{1}$. When testing for similarity, it means that there is something to compare: one takes two or more entities, compares them property $\&$ value by property $\&$ value and calculate their closeness with metrics common in data mining and clustering. Analyses of similarity-from [16] to [2] —are, like clustering algorithms, useful for grouping instances, but is limited to being similar with respect to one or a few values of properties only. If the property chosen for similarity matching is not a sortal property (the analogue of a count noun), then no classification occurs sensu semantics of the set-as in ontologies, e.g., classification of proteins based on properties instead of measured values [17]—but only an ad hoc grouping of the entities. The latter can be useful for extracting a tree to classify the data afterward where one can condense the resulting tree as one desires (e.g., hierarchical organisation of protein clusters from SwissProt and TrEMBL [18]). However, the two operations are distinct. For instance, clustering objects by being red and others purple does not reveal anything about the identity of the objects in the sets for Red and Purple. A combination of the measurement \& semantics approach is the notion of guilt-by-association (GBA) in mRNA expression analyses, where uncharacterised genes may share the same functional roles as already annotated genes in the same cluster. It combines the values of colours on the microarray with the universals represented in the Gene Ontology [19]; thus, using both quantitative and qualitative granularity to achieve meaningful clustering (see also [20] for many more such combined similarity measures). We can define similarity with respect to granularity as follows, where $P T$ stands for particular and $U$ for universal.

\footnotetext{
${ }^{1}$ In the remainder of the article we use 'granule' and 'granular level' interchangeably. They merit more precise definitions that hold across the various subdisciplines grouped under granular computing, but this is beyond the scope of the article.
} 
Definition 1 (Similarity $\sigma$ ): Let $x, y, \ldots \in P T$ or $x, y, \ldots \in$ $U, \gamma_{i}$ a granule (granular level), gran_of $\left(x, \gamma_{i}\right)$ and gran_of $\left(y, \gamma_{i}\right)$, then $\gamma_{i}$ contains $\{x, y, \ldots\}$ that are similar to each other, $x \sigma y$ in $\gamma_{i}$, with respect to quality property, $p_{q}$, with measurable values that are within the defined value space $\varepsilon_{x}$ valid for $p_{q}$ of $x . \sigma$ is reflexive, symmetric, and transitive.

Observe that in a setting without granularity-i.e., omitting the assertion that granule $\gamma_{i}$ contains $x, y$-one cannot prove symmetry and transitivity, because it is possible that $\varepsilon_{y}<$ $\varepsilon_{x}$ such that the particular value of $x$ is beyond $\varepsilon_{y}$. And if $x \sigma y$ holds when the value of $y$ for $p_{q}$ is within $\varepsilon_{x}$, then this does not imply that if $y \sigma z$ (because $z$ 's value for $p_{q}$ is within $\varepsilon_{y}$ ) then $z$ 's value still falls within $\varepsilon_{x}$; transitivity can hold, but not necessarily for all entities. In contrast, in the granular setting a) $\varepsilon$ is set for each granule, hence $\sigma$ is symmetric and transitive with respect to the objects residing in the same granule, and b) if $\varepsilon_{y}<\varepsilon_{x}$ (according to the source data or granule specification) such that the particular value of $x$ is beyond $\varepsilon_{y}$ then it must be that $y$ resides in finer-grained level of granularity compared to the granule where $x$ resides.

\section{INDISTINGUISHABILITY AND EQUIVALENCE}

Given similarity, it is easier to highlight the distinction with indistinguishability that concerns objects being indistinguishable in some coarse $\gamma_{i}$, where one cannot compare and determine if objects are similar or not. It assumes that at a finer $\gamma_{j}$ the entities are distinguishable from each other. The difference between similarity and indistinguishability is demonstrated for coffee, cholera, and whooping cough in the example, and analysed afterward regarding, respectively, qualitative and quantitative aspects of granulation.

Example Take the meaning of the statement "caffeine and cholera toxin both cause prolonged activation of the Second Messenger System (SMS)", then the effects of both caffeine and the cholera toxin are similar enough with their "prolonged activation" to be put together at this $\gamma_{i}$, where finer-grained mechanisms of prolongation of SMS, if any, are indistinguishable from each other at $\gamma_{i}$. This is different from claiming both mechanisms are 'exactly similar', the same, because based on the given information, one cannot prove either way. In fact, at a finer-grained $\gamma_{j}$, the mechanisms are distinguishable and found to be distinct, thus not similar enough to be grouped together. More precisely: caffeine inhibits phosphodiesterase activity that otherwise would break down cAMP, whereas the A subunit of the cholera toxin binds to the $G$ protein, thereby impairing $G$ to return to its inactive state and locking $G$ into its active form, resulting in excessive production of cAMP. Thus, they are distinguishable with regards to the properties involved, hence looking for similarity values is irrelevant in the comparison.

The pertussis toxin, produced by the causative agent of whooping cough, Bordetella pertussis, also interferes with a $\mathrm{G}$ protein in the SMS but not the same one as the cholera toxin does. The stimulatory $\mathrm{G}_{s}$ protein is the target of cholera toxin and the inhibitory $\mathrm{G}_{i}$ protein is the target of the pertussis toxin. $\mathrm{G}$ proteins are signal-coupling proteins with similar structural and functional motifs: $G_{s}$ and $G_{i}$ have the same $\beta$ and $\gamma$ subunits but have different $\alpha$ subunits. Thus, the same property is involved but has distinct values; hence, the $\alpha$ subunits of $\mathrm{G}_{s}$ and $\mathrm{G}_{i}$ can be compared on their similarity (3D shape, aminoacid sequence). When both are categorised as whole G protein they are indistinguishable; it is only at the finer-grained level where one has 'access' to the properties of its structural components that one can distinguish them. $\diamond$

This example illustrates two key issues about indistinguishability: being (in-)distinguishable and how one moves from distinguishable to indistinguishable (and back). The former involves properties, the latter makes use of multiple types of relations to make things (in-)distinguishable, which needs to be disambiguated. The first step to clarify this, is to analyse the indistinguishability relation as introduced by Hobbs [9] and followed up by [10], [11]: this indistinguishability relation " $\sim$ " between $x$ and $y$, says that $x$ and $y$ are indistinguishable if no relevant predicate distinguishes between them, where $p$ is some predicate and $\mathbf{R}$ a subset of predicates of a first order logical theory $T_{0}(1)$.

$$
x \sim y \leftrightarrow(p \in \mathbf{R})(p(x) \equiv p(y))
$$

Analysing (1) and the descriptions of the "indistinguishability" relation [9]-[11], it is clear that the axiom is another rendering of the common equivalence relation:

Definition 2 (Equivalence relation): Let $X$ be a set, and $R$ a binary relation on $X$ that is a subset of $X \times X$. The equivalence relation $\sim$ (alternative notation: $\equiv)$ is a special case of $R$ : an equivalence relation on $X$ is a binary relation on $X$ such that: $x \sim x$ for all $x \in X$ (reflexivity), if $x \sim y$ then $y \sim x$ for all $x, y \in X$ (symmetry), and if $x \sim y$ and $y \sim z$ then $x \sim z$ for all $x, y, z \in X$ (transitivity).

With this general definition, one can then define a particular equivalence relation: to state that $x$ and $y$ are equivalent under a given condition, we have, e.g., $x \sim y$ iff $x \equiv y(\bmod$ 2 ), meaning that $x$ and $y$ have the same value after $y$ is divided by 2 ; concerning $(1)$, the " $(\bmod 2)$ " is replaced by an arbitrary predicate $p$. For instance, [7] use the equivalence relation in the context of rough sets (addressed in the next section), whereas [21] limits the equivalence relation to equal durations of particular temporal intervals. One should be able to extended this equivalence relation also to other subject domains, like that we can say that compatible transplant kidneys are equivalent at an Organ-granule, even though its cells have different characteristics (cell surface proteins, DNA sequence, etc.). This cannot be represented as such with just the equivalence relation, due to the lack of reference to a property and the absence of the qualifier 'given $\gamma$ '.

Another issue is the differences in intended semantics and usage of the equivalence and indistinguishability relations. For instance, say, if $X$ is a set of toys that has to be partitioned and 
one defines $\sim$ as has the same colour as, then a subset $Y$ of $X$ for the colour blue has as members all blue toys; but the toys still can be identified. On the other hand, taking the example above with $\mathrm{G}_{s}$ protein, $\mathrm{G}_{i}$ protein $\in Y$, G protein $\in X$, then $\mathrm{G}_{s}$ and $\mathrm{G}_{i}$ are indistinguishable in $X$ for properties function and $\alpha$-subunit; hence, either $X$ or $Y$ provides the identity criterion to identify objects. Summarizing:

* The equivalence relation has a 'conversion parameter' to make $y$ equivalent to $x$ with respect to that parameter and it focuses on partitioning sets - that is, making objects in $X$ distinguishable in subsets of $X$;

* Before, during, and after creating disjoint subsets with the equivalence relation, one still can distinguish - identify - the objects: identity or identification of the objects is already provided by membership of $X$.

* Indistinguishability aims to be more generic to any kind of property (although this is not excluded with the equivalence relation), including qualitative properties and it focuses on the direction from similar and distinguishable in $Y$ toward indistinguishable-i.e., the opposite of partitioning: a unifying property among objects to make them indistinguishable from each other in set $X$.

* With indistinguishability, one cannot distinguish between the objects at their coarsergrained level: the objects' identity criterion lies with the characteristics of the finer-grained set $Y$, not with the unified set $X$ at the coarser-grained level.

Of course, $\sim$ itself does not say anything about the direction of usage - partitioning or unifying - but the semantics of indistinguishability is more comprehensive. To push it further and clarify subtle, but essential, differences, we have to introduce Hobbs' (and others) simplification function, $\kappa$, and articulation first; that is, on how one moves from distinguishable to indistinguishable and back. The simplification function collapses a theory $T_{0}$ into a coarse-grained simpler theory $T_{1}$ : $x \sim y \equiv \kappa(x)=\kappa(y) ; \kappa$ is undefined but constrained by if $x$ is at a finer level than $y$ then its mapping does not end up in a higher level than to which $y$ maps into, more precisely: $\neg(\exists x, y)(x<y \wedge \kappa(x)>\kappa(y))$ [9]. Thus, if we have two finegrained entity types $A$ and $B$ (or their respective instances a and b) in $\gamma_{j}$, with two mappings $\kappa(\mathrm{A}) \rightarrow \mathrm{C}$ and $\kappa(\mathrm{B}) \rightarrow \mathrm{C}$ and $\mathrm{C}$ is an entity type in the coarse-grained $\gamma_{i}$, and $\gamma_{j} \prec \gamma_{i}$ (and not $\gamma_{j} \prec \gamma_{x} \prec \gamma_{i}$ ), then $A \sim B$ at level $\gamma_{i}$. Substituting for the $G$ proteins in the example, one has $A=G_{s}$ Protein, $B=G_{i}$ Protein, and $\mathrm{C}=\mathrm{G}$ Protein, or, as Hobbs and later also [10] put forward, a time interval in $T_{0}$ maps into an instant in coarser-grained $T_{1}$. The inverse procedure of simplification is for distinguishing between $x$ and $y$, what Hobbs calls articulation, that has the aim to find (partial) predicates wherein $x$ and $y$ differ, which corresponds to partitioning. For substance $p$ that has an $~$ determined by the granule of $p$, then "a piece of $p$ has proper parts which are of the same substance, provided it has two distinguishable points" (emphasis added) [9]:

$$
\begin{array}{r}
\forall x\left(p(x) \wedge \exists y_{1}, y_{2}\left(i n\left(y_{1}, x\right) \wedge i n\left(y_{2}, x\right) \wedge y_{1} \nsim y_{2}\right) \supset\right. \\
\exists z(\text { part_of }(z, x) \wedge z \neq x \wedge p(z)))
\end{array}
$$

Continuing now with the equivalence vs. indistinguishability, there are three issues regarding simplification and articulation with respect to granularity. First, the formalisation and semantics of articulation is not the inverse of simplification. During simplification, one discards a property in the same way as with specialisation/generalisation, but with articulation there is 'something' $z$ new introduced, that is part of the known property $x$. One goes up to a coarser granule (coarsergrained level) by discarding a property and returning down by only identifying a part of a known property that does not necessarily reintroduce the discarded property, except if the property is a compound property and meets the requirement of compositionality. In casu, using aforementioned simplification from time interval to instant [9], [10], then articulating from instant to interval introduces several issues: how is the interval part of the instant, and, more importantly, what are the two distinguishable parts of the same type of the interval? This only can be if the interval itself is split-up in parts to be able to distinguish between, say, the 3rd second and the 5th second in an interval of $>5$ seconds, which is at a yet finer-grained level of granularity than the interval. Of course, it is different when we look at a sequence of events or processes occurring during the interval instead of the interval itself; then we have a straight-forward case of whole process and its process-parts, but this does not imply that the process-parts are of the same type as Hobbs' defined in (2).

Second, the generic notion of being indistinguishable represents a coarse-grained meaning of what is indistinguishable: it lumps together being indistinguishable caused by subtyping, structural parts, sub-processes, spatial parts, measurement scales and so forth. Further, by considering only an arbitrary property of some object, $p(x)$, one can randomly discard and add properties, but ignoring something at the same level of granularity because subjectively it has nothing to do with the domain is distinct from granularity itself. In contrast stands the use of a non-arbitrary, essential, identity criterion-providing property to make something indistinguishable: having lost the identity criterion at the coarser-grained granule has made those objects as they are in the finer-grained granule non-identifiable as such in the coarser-grained one. Analogously, partitioning with the equivalence relation according to an essential identity criterion-providing property generates not just new subsets, but, unlike the Blue toys mentioned above, those subsets are the set extensions of universals, hence, sets with meaning.

The third problem follows from the first and second: the underspecified indistinguishability relation (equivalence relation) and the two operators do not characterise a granule, let alone granularity, except for highlighting it has something to do with properties. Summarizing the qualitative aspects of indistinguishability, we have an indistinghuishable-ising property, an implicit distinction between using either properties or their values for granulation, involvement of coarse- and finergrained granules, and a notion of the resulting fine-/coarsegrained sub-/super-set if it should be a set extension of a universal. This still lacks the integration of quantitative aspects for indistinguishability. Concerning indistinguishability 
for measurements or scale-based granules, we have to address in more detail a variant of indistinguishability, better known as the indiscernibility relation, which is discussed next.

\section{A. Indiscernibility}

An aspect of indistinguishability is indiscernibility: the property under consideration is the same, but some measurable, numerical value, of the property is indistinguishable at a coarser granule. Approaches to indiscernibility are summarised here and cast in relation to the preceding section.

The simplest approach is [9]'s "measure of undefinedness", $\varepsilon$, where $f$ is a restricted case of the simplification function $\kappa$ for indistinguishability for measurement values: $x \sim y \equiv$ $\kappa(x)=|f(x)-f(y)|<\varepsilon$. For instance, real numbers are rounded off to its nearest integer, such that two reals, say, 11.1 and 11.2, at level $\gamma_{j}$ are indistinguishable at its higher level of integers, $\gamma_{i}$, where both reals map into the integer 11 . Independently, this idea has been developed to a much larger, and usable, scope in the research areas of rough sets, its rough mereological approach, approximation spaces of similarity [4]-[6], and fuzzy sets [1], [22] with "fuzzified equality" [23] where some instance is part of a set to a degree.

Going into some detail for the approximation spaces and rough mereological approach [5], let Ind be the indiscernibility relation, $U$ a set of objects, $P(U)$ its power set, $\mathcal{N}$ an uncertainty function defined on $U$ where $\mathcal{N}(x)$ is a neighbourhood of object $x$, and $\nu$ the inclusion function defined on $P(U) \times$ $P(U)$ that measures the degree of inclusion of sets, then we can define the two operations for lower $\left(A S_{l}\right)$ and upper $\left(A S_{u}\right)$ approximation: $A S_{l}(X)=\{x \in U: \nu(\mathcal{N}(x), X)=1\}$ and $A S_{u}(X)=\{x \in U: \nu(\mathcal{N}(x), X)>0\} . \mathcal{N}(x)$ can be defined by the indiscernibility relation Ind in two ways, either as equivalence relation or as tolerance (or similarity) relation $\tau$, where $\tau \subseteq U \times U$ (in [5]'s terminology). If the former, then $\mathcal{N}(x)=[x]_{\text {Ind }}$ where the equivalence class $[x]$ comprises $x$ and its neighbourhood, if the latter, we have $\mathcal{N}(x)=\{y \in$ $U: x \tau y\}$ and thereby making $\mathcal{N}$ equal to the tolerance class $\tau$ defined by $x$. Thus, $\tau$ is conceptually equivalent to the equivalence relation $\sim$ of the previous section. In addition, $y$ is near enough to $x$-similar, $y \sigma x$ - to be grouped together because they are in the same approximation space $A S$ and both objects are included thanks to the inclusion function $\nu$; $A S$ is then defined by $A S=(U, \mathcal{N}, \nu)$ [5]. Approximation operations-thus also classifying the object into a particular granule — can be adjusted via parametrisation of $\mathcal{N}$ and $\nu$. Note that $\nu$ can be generalised to the rough mereological approach with the inclusion relation $\mu_{r}$ such that $x \mu_{r} y$ denotes that $x$ is part of $y$ to a degree $r$. Thus, set membership can be determined if the value of a property falls within the defined lower and upper bound for that set. [23] use $\mu$ to denote fuzzification of set membership $\epsilon$, represented as an element $x$ that has a membership degree $\mu(x)$ to a fuzzy set $\mu$ with the usual further specifications for degrees of membership in fuzzy sets, like taking into account $\varepsilon$ for closeness to a point (as Hobbs did as well), and minimum \& maximum values. This lower-upper bound usage matches the sieve conceptualisation of [12].

These specifications, which omit direct reference to granules (or levels of granularity, levels of abstraction), permit two orthogonally positioned usages. One concerns the value or value range for each granule within a granulation hierarchy, such as $\mathrm{km}^{2}$ and $\mathrm{m}^{2}$, and the other the precision of each specific level, or the refinement of measurements for the granule, such as " $\mathrm{km}^{2} \pm 1 \mathrm{~m}^{2}$ " or " $\mathrm{km}^{2} \pm 1 \mathrm{~cm}^{2}$ ", where the choice for $\mathrm{m}^{2}$ or $\mathrm{cm}^{2}$ is provided by the approximation space that covers both intended and enforced indistinguishability ${ }^{2}$. Examples of degrees of membership are properties such as colour shades, but this is rarely used as a criterion for granulation of universals (although it can be used as an indirect means [19], [20]). It is important to stress the difference between the complementary granularities with indiscernibility: the primary 'axis' involves the scale relevant for a particular granulation hierarchy and the secondary axis the refinement, amount of impreciseness, that is given in any of the finergrained quantities at each level.

\section{INDISTINGUISHABILITY FOR GRANULATION}

Having addressed both the qualitative and quantitative aspects of indistinguishability, we can proceed to a definition of indistinguishability that takes into account both quantitative and qualitative granularity, where $U$ stands for universal, and $P T$ particular, $Q$ quality, and $V$ region, which are DOLCE foundational ontology categories [24]:

Definition 3 (Indistinguishability): Let $x$ and $y$ reside in granule $\gamma_{j}$, there exists a $\gamma_{i}$ s.t. $\gamma_{j} \prec \gamma_{i}$ contained in one hierarchy of granules, $z$ resides in $\gamma_{i}, x$ and $y$ map into $z$, and $z \in P T$ or $z \in U$, then $x$ and $y$ are $\varphi$-indistinguishable from each other, $x \approx y$, at $\gamma_{i}$ with respect to $z . \varphi$ denotes the type of (functional or not functional) relation that relates $x, y$ to $z$, its distinguishing property, property value, or value range between the two levels, i.e., $\varphi \in \mathcal{P}$ or $\varphi \in V$, where $\mathcal{P}$ is the set of binary predicates $P$ on the domain $(P T \times P T$ or $U \times U), \approx \neq \varphi$, and $V$ is the set of declared values and value ranges for property $Q$ ).

Observe the difference with the equivalence relation regarding embedding indistinguishability in the framework of granularity and inclusion of a reason for indistinguishability. With $\varphi$, one has the freedom to choose how objects are (made) indistinguishable, and to record this for each granulation hierarchy in a software application. For instance, for qualitative granularity, the $\varphi$ of $\varphi$-indistinguishable with respect to set-based granularity then refers to exactly that additional property of the subsumed entity type compared to its subsumer, whereas with whole-part granulation the $\varphi$ refers to the very fact that the finer-grained entity type is part-indistinguishable.

2 [23] add distinctions for "enforced" and "intended" indistinguishability, which affects representation of the UoD and its usage in software systems, but not a domain- and implementation-independent definition. The former is caused by limited precision due to noisy data, the equipments itself and indirect measurement taking, the latter corresponds to impreciseness because the measurement-taker does not care about more precise measurements. 
Modifying the definition of the equivalence relation by adding reference to granules is also an option: [4] takes steps in that direction with a "coarse-grained universe" given an equivalence relation $E$ on universe $U$, but he still permits partitioning into arbitrary sets or named subsets. Although a name still can be a mere label, it is a step toward meaningful subsets as opposed to an arbitrary grouping of objects. To have subject domain semantics for indistinguishability at the ontological level, the sets resulting from partitioning or unifying should be the set-extensions of universals, which can be met with $\varphi \in \mathcal{P}$ and the reference to particulars and other universals. However, the equivalence relation has a widespread use beyond granular computing and therefore may result in confusion if the definition is modified, whereas the notion of indistinguishability remains mainly within the context of granulation and therefore is easier to amend.

With the three definitions, it follows that there must be at least two granules in a granulation hierarchy (proven below). This contradicts Salthe [14], [15], who asserts that there must be at least three levels to always have one level above and one level below the level of interest. However, there is neither necessarily an infinite chain of granulation steps [12] nor is this inherently demanded from any of the well-established notions of indistinguishability, partitioning, and equivalence: the only requirement these relations imply is to have one level above or one level below the level of interest. We formally prove this in Theorem 1 below. From this proof it follows that there is a strict order among the granules in any particular hierarchy of granules (Lemma 4), i.e., which are created through applying the same criterion such as parthoodbased or metric scale-based, and therefore granules in such a hierarchy must be disjoint (Corollary 1). To arrive at these results, we first have to take the definitions of equivalence, similarity, and indistinguishability, and prove exclusion of coexisting similarity and indistinguishability in Lemma 1 , that indistinguishability is a subtype of the equivalence relation (Lemma 2), and its properties in Lemma 3.

Lemma 1: If $x$ and $y$ are similar, $x \sigma y$, in some granule, then they cannot also be indistinguishable, $\neg(x \approx y)$.

Proof: Given Definition 1, x,y, and $x \sigma y$, this implies that at least their value spaces $\varepsilon_{x}$ and $\varepsilon_{y}$ must either overlap or overlap properly to ensure $y$ falls within $\varepsilon_{x}$.

First, using overlap in General Extensional Mereology [25], then overlap $\left(\varepsilon_{x}, \varepsilon_{y}\right)$ holds iff $\exists z$ part_of $\left(z, \varepsilon_{x}\right) \wedge$ part_of $\left.\left(z, \varepsilon_{y}\right)\right)$. Assume $x \approx y$, which implies $\varepsilon_{x} \approx \varepsilon_{y}$. Substituting $\varepsilon_{y}$ for $\varepsilon_{x}$ (or vv.), collapses into identity $\left(\varepsilon_{x}=\varepsilon_{y}\right)$ for $p_{q}$ and thereby contradicting overlap. Take $\varepsilon_{x}$ for $x$, then $x$ must have been identified prior to determine $\varepsilon_{x}$, because $p_{q}$ is a property of $x$. Given $x$, one takes another object $y$ to measure $p_{q}$, which is known to be $\neg x$, thereby contradicting $x \approx y$. (Note: if the measured values are the same, then $x$ and $y$ are $100 \%$ similar, because $x$ and $y$ were already identified, hence not $(x \approx y)$.).

Second, consider proper overlap, p_overlap $\left(\varepsilon_{x}, \varepsilon_{y}\right)$, which holds iff overcoss $\left(\varepsilon_{x}, \varepsilon_{y}\right) \wedge \neg\left(\operatorname{overcoss}\left(\varepsilon_{y}, \varepsilon_{x}\right)\right)$, with overcross defined as

$$
\operatorname{overcross}(x, y) \triangleq \operatorname{overlap}(x, y) \wedge \neg \text { part_of }(x, y) \text {. }
$$

Substitution for the overcross relations in p_overlap $\left(\varepsilon_{x}, \varepsilon_{y}\right)$ and then substitution for overlap gives

$$
\left(\exists z\left(\text { part_of }\left(z, \varepsilon_{x}\right) \wedge \text { part_of }\left(z, \varepsilon_{y}\right) \wedge \neg \text { part_of }\left(\varepsilon_{x}, \varepsilon_{y}\right)\right)\right) \wedge
$$$$
\neg\left(\exists z\left(\text { part_of }\left(z, \varepsilon_{y}\right) \wedge \text { part_of }\left(z, \varepsilon_{x}\right) \wedge \neg \text { part_of }\left(\varepsilon_{y}, \varepsilon_{x}\right)\right)\right)
$$

push negation within the braces in the second part:

$\left(\exists z\left(\right.\right.$ part_of $\left(z, \varepsilon_{x}\right) \wedge$ part_of $\left(z, \varepsilon_{y}\right) \wedge \neg$ part_of $\left.\left.\left(\varepsilon_{x}, \varepsilon_{y}\right)\right)\right) \wedge$ $\left(\neg \exists z\left(\right.\right.$ part_of $\left(z, \varepsilon_{y}\right) \wedge$ part_of $\left.\left(z, \varepsilon_{x}\right)\right) \vee \neg\left(\neg\right.$ part_of $\left.\left.\left(\varepsilon_{y}, \varepsilon_{x}\right)\right)\right)$ Either the first quantification " $\exists z()$ " or the second one with the negation has to be false, the latter can be false but then $\neg\left(\neg\right.$ part_of $\left.\left(\varepsilon_{y}, \varepsilon_{x}\right)\right)$ must be true (thanks to the $\vee)$. With elimination of the double negation as well, then $\left(\exists z\left(\right.\right.$ part_of $\left(z, \varepsilon_{x}\right) \wedge$ part_of $\left(z, \varepsilon_{y}\right) \wedge \neg$ part_of $\left.\left.\left(\varepsilon_{x}, \varepsilon_{y}\right)\right)\right) \wedge$ part_of $\left(\varepsilon_{y}, \varepsilon_{x}\right)$

holds. The combination of $\neg$ part_of $\left(\varepsilon_{x}, \varepsilon_{y}\right)$ and part_of $\left(\varepsilon_{y}, \varepsilon_{x}\right)$ is proper parthood, hence, p_part_of $\left(\varepsilon_{y}, \varepsilon_{x}\right)$, which implies $\varepsilon_{x} \neq \varepsilon_{y}$, and in turn $\neg\left(\varepsilon_{x} \approx \varepsilon_{y}\right)$. Given that the respective spaces of $x$ and $y$ can be distinctly identified, $x$ and $y$ must be distinguishable, hence $\neg(x \approx y)$.

Lemma 2: If $x$ and $y$ are indistinguishable, $x \approx y$, then they are also equivalent, $x \sim y$.

Proof: Given Definition 2, we have $\forall x, y(x \sim y \rightarrow$ $R(x, y)$ ), where $R \subseteq S \times S$, and $x \sim y$ with $x, y \in S$, for any arbitrary condition $c \in C$. Given Definition 3, we have $\forall x, y(x \approx y \rightarrow R(x, y))$, where $R$ is a subset of the contents $S$ of a granule, $S \times S$, but $x \approx y$ (with $x, y \in S$ ) is restricted to $\varphi$ type of conditions s.t. $\{\varphi\} \subset C$; hence, $\forall x, y(x \approx y \rightarrow x \sim y)$.

Lemma 3: The indistinguishability relation $\approx$ has the metaproperties of reflexivity, symmetry and transitivity.

Proof: From Lemma 2 we know that $\forall x, y(x \approx y \rightarrow$ $x \sim y$ ), therefore $\approx$ inherits the properties of $\sim$. Given Definition 2, we know that $\sim$ has the meta-properties of being reflexive, symmetric, and transitive, therefore $\approx$ is also reflexive, symmetric, and transitive.

Theorem 1: A granulation hierarchy $H$ must contain at least two granules $G$, formally:

$\forall x\left(H(x) \rightarrow \exists^{\geq 2} y(\operatorname{contains}(x, y) \wedge G(y))\right)$

Proof: Given Definition 2 and Definition 3, we have $x, y, \ldots \in X, R$ defined over $X \times X, \mathcal{R}$ set of relations, $\forall x, y(x \sim y \rightarrow R(x, y))$ and $\forall x, y(x \approx y \rightarrow R(x, y))$.

1. Assume one level: By Lemma 1, we cannot have one set (granule) where both $x \sigma y$ and $x \approx y$ hold, therefore granulation results in $>1$ level.

2. Assume at least one level: suppose $\forall x, y(x \approx y \leftrightarrow$ $R(x, y))$, i.e. $\{\approx\} \equiv \mathcal{R}$, then all objects in contained in $\gamma_{i}, X$ (i.e., gran_of $\left(X, \gamma_{i}\right)$ ), are indistinguishable and $\{x, y\} \equiv X$. But by Definition 3, we have $\approx \neq \varphi$ and $\varphi \in$ $\mathcal{R}$, therefore at least $\approx, \varphi \in \mathcal{R}$, therefore there must exist a subset $Y$ s.t. $x, x^{\prime} \in Y, \neg\left(x \approx x^{\prime}\right)$ (thus also $x^{\prime} \neq y$ ), hence $Y \subset X$ and gran_of $\left(Y, \gamma_{i}^{\prime}\right)$, i.e. $\geq 2$ levels.

3. Assume at least three levels, ... $\prec \gamma_{j} \prec \gamma_{i} \prec \gamma_{h} \prec \ldots$, 
then $x$ in focal $\gamma_{i}$ must be related to at least two entities, $y$ in $\gamma_{j}$ and $z$ in $\gamma_{h}$, which requires a ternary $R(x, y, z)$ to span 3 granules. $\sigma, \sim, \approx$, and $\varphi$ are binary relations and by point $1 \& 2$ above, already permit granulation, thereby contradicting a minimum of 3 granules.

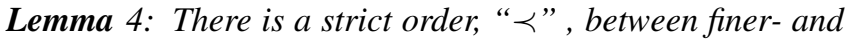
coarser granules in a granulation hierarchy.

Proof: Follows from Lemma 1 and Theorem 1 (nr.2).

Corollary 1: Within one granulation hierarchy, no two granules are the same.

With these results we can define granulation hierarchy:

Definition 4 (Granulation hierarchy): Let $X$ and $Y_{i}$ denote sets $(i \geq 1)$ in universe $\Delta, Y_{i} \subset X$, then a granulation hierarchy $H \in \mathcal{H}$ is the ordered sequence of granules (levels of granularity) $\gamma_{1}, \ldots, \gamma_{n} \in \Gamma$ (with $n \geq 2$ ), $\gamma_{n} \prec \gamma_{n-1} \ldots \gamma_{2} \prec$ $\gamma_{1}$, obtained from successively either partitioning $X$ into subsets $Y_{i}$ or making objects in $Y_{i} \varphi$-indistinguishable in $X$, and gran_of $\left(X, \gamma_{i-1}\right)$ and gran_of $\left(Y_{i}, \gamma_{i}\right)$.

Observe that this permits creation of multiple hierarchies (views, granular perspectives) within a demarcated universe, so that each hierarchy has its own criterion by which its contents is granulated, such as with cartographic maps of different detail or parthood.

\section{CONCLUSiOns}

Indistinguishability was disambiguated and compared with the well-known equivalence and similarity relations. For objects being similar, one has some measurable property to compare the objects, whereas indistinguishable objects cannot be compared regarding the focal property. Indistinguishability starts from distinguishable objects at a finer granule and is used to move 'up' toward being indistinguishable, whereas the equivalence relation starts from some set and generally is used to partition objects into finer-grained sets.

Indistinguishability influences the conceptualization of granule concerning how properties are introduced and removed going from a coarse to a finer-grained granule and vv, which implies that finer granules have more or more precisely specified properties and values compared to their coarsergrained version higher up in the granulation hierarchy. We defined indistinguishability and a basic version of granulation hierarchy; proved reflexivity, symmetry, and transitivity of indistinguishability; and that any granulation hierarchy must contain at least 2 granules (levels of granularity) which relate through a strict order. This may aid consistency in usage of granulation in software applications through implementation of the properties as constraints and the granulation hierarchy as additional structuring frame for organising granules.

We are currently investigating how these results can be used for defining complex granular queries to enhance information retrieval from databases and ontologies.

\section{REFERENCES}

[1] L. A. Zadeh, "Toward a theory of fuzzy information granulation and its centrality in human reasoning and fuzzy logic," Fuzzy Sets and Systems, vol. 90, no. 2, pp. 111-127, 1997.

[2] C. Mencar, G. Castellanoa, and A. M. Fanellia, "Distinguishability quantification of fuzzy sets," Information Sciences, vol. 177, no. 1, pp. 130-149, 2007.

[3] Y. Hata and M. Mukaidono, "On some classes of fuzzy information granularity and theirrepresentations," in Proceedings of the 29th IEEE International Symposium on Multiple-Valued Logic, 1999, pp. 288-293.

[4] Y. Y. Yao, "A partition model of granular computing," LNCS Transactions on Rough Sets, vol. 1, pp. 232-253, 2004.

[5] J. F. Peters, A. Skowron, S. Ramanna, and P. Synak, "Rough sets and information granulation," in Proceedings of 10th International Fuzzy Systems Association World Congress, ser. LNAI, T. Bilgic, B. Baets, and O. Kaynak, Eds., vol. 2715. Springer-Verlag, 2002, pp. 370-377.

[6] A. Skowron and J. F. Peters, "Rough sets: Trends and challenges plenary paper," in Proc. of RSFDGrC 2003: Rough Sets, Fuzzy Sets, Data Mining, and Granular Computing, ser. LNAI, G. Wang, Q. Liu, Y. Yao, and A. Skowron, Eds., vol. 2639. Springer, 2003, pp. 25-34.

[7] T. Bittner and J. Stell, "Stratified rough sets and vagueness," in Spatial Information Theory. Cognitive and Computational Foundations of Geographic Information Science (COSIT'03), W. Kuhn, M. Worboys, and S. Timpf, Eds., 2003, pp. 286-303.

[8] Y. H. Chen and Y. Y. Yao, "Multiview intelligent data analysis based on granular computing," in IEEE International Conference on Granular Computing (GrC2006), vol. 1. IEEE Xplore, 2006, pp. 281-286, 1.

[9] J. R. Hobbs, "Granularity," in Proc. of IJCAI85, 1985, pp. 432-435.

[10] I. Mani, "A theory of granularity and its application to problems of polysemy and underspecification of meaning," in Proc. of KR'98, A. Cohn, L. Schubert, and S. Shapiro, Eds. San Mateo: Morgan Kaufmann, 1998, pp. 245-255.

[11] G. I. McCalla, J. E. Greer, B. Barrie, and P. Pospisil, "Granularity hierarchies," Computers and Mathematics with Applications: Special Issue on Semantic Networks, vol. 23, pp. 363-376, 1992.

[12] C. M. Keet, "A taxonomy of types of granularity," in IEEE International Conference on Granular Computing (GrC2006), vol. 1. IEEE Xplore, 2006, pp. 106-111, 1.

[13] Y. Y. Yao, "The art of granular computing," in Proc. of the Int.l Conf. on Rough Sets and Emerging Intelligent Systems Paradigms, 2007.

[14] S. Salthe, Evolving hierarchical systems: their structure and representation. New York: Columbia University Press, 1985.

[15] - "Summary of the principles of hierarchy theory," http://www.nbi.dk/ natphil/salthe/hierarchy_th.html, November 2001.

[16] P. Gärdenfors, Conceptual Spaces: The Geometry of Thought. Bradford Books: MIT Press, 2000

[17] K. Wolstencroft, P. Lord, L. Tabernero, A. Brass, and R. Stevens, "Using ontology reasoning to classify protein phosphatases," in 8th BioOntologies Meeting 2005, Detroit, USA, 24 June 20052005.

[18] N. Kaplan, O. Sasson, U. Inbar, and et al, "Protonet 4.0: A hierarchical classification of one million protein sequences," Nucleic Acids Research, vol. 33, pp. D216-D218, 2005.

[19] Y. Zhou, J. A. Young, A. Santrosyan, and et al., "In silico gene function prediction using ontology-based pattern identification," Bioinformatics, vol. 21, no. 7, pp. 1237-1245, 2005.

[20] A. Bender and R. C. Glen, "Molecular similarity: a key technique in molecular informatics," Org. Biomol. Chem., vol. 2, pp. 3204-3218, 2004.

[21] H. R. Schmidtke, "Granularity as a parameter of context," in Proc. of CONTEXT 2005, ser. LNCS, A. Dey, B. Kokinov, D. Leake, and R. Turner, Eds., no. 3554. Springer, 2005, pp. 450-463.

[22] M. Reformat, W. Pedrycz, and N. Pizzi, "Building a software experience factory using granular-based models," Fuzzy Sets and Systems, vol. 145 pp. 111-139, 2004.

[23] F. Klawonn and R. Kruse, "The inherent indistinguishability in fuzzy systems," in Logic versus Approximation: Essays Dedicated to Michael M. Richter on the Occasion of his 65th Birthday, ser. LNCS, W. Lenski, Ed. Springer: Berlin / Heidelberg, 2004, vol. 3075.

[24] C. Masolo, S. Borgo, A. Gangemi, N. Guarino, and A. Oltramari, "Ontology library," WonderWeb Deliverable D18 (v1.0), 122003.

[25] A. C. Varzi, "Mereology," in Stanford Encyclopedia of Philosophy, fall 2004 ed., E. N. Zalta, Ed. Stanford, 2004, http:// plato.stanford.edu/archives/fall2004/entries/mereology/. 